ФТИ-2020

\title{
X-RAY IMAGE SEGMENTATION WITH THE USE OF MACHINE LEARNING ALGORITHMS
}

\author{
Artemyev M.S. ${ }^{1}$, Serazetdinov A.R. ${ }^{2}$, Smirnov A.A. ${ }^{1}$ \\ 1) Ural Federal University, Yekaterinburg, Russia \\ 2) National Research Nuclear University MEPhI, Moscow, Russia \\ E-mail: mihailartemev90647@gmail.com
}

Oncological diseases interpretation through CT imaging is a commonly used method of cancer issues diagnosis. Designed neural network reaches aggregations segmentation accuracy of about $90-93 \%$, although some weak points still remain.

Breast cancer has been long concerned as one of the most significant counterparts of common cancer problem. This cancer reveals itself with heart tumors or aggregations. Recognition of malignant tumors in the early stages is rather beneficial as it could provide the patient with necessary medicine in due course to slow down and remedy the disease. The analysis is done in most of the cases through X-ray images processing due to the relatively low cost of the method and for some save reasons.

Although X-ray image processing is commonly done through expert diagnostics, the automation is still needed to increase effectiveness and reduce costs. This could be conducted through convolutional neural network use to distinguish the tumors.

The current state of cancer segmentation with state-of-the-art neural networks has already gained efficiency of about $97 \%$. Although these figures are quite beneficial from the laboratory point of view, in practical cases the problem of bad image quality frequently arises. X-ray modules used in many facilities still lack of directly needed shape peculiarities, so the standard solutions trained on laboratory images are considered as impractical in some cases.

On the other hand, the amount of works being done on these topics is still growing, so systematization is required, and problem focusing is needed.

The review of commonly described and some distinct neural networks used for breast cancer recognition is presented, underlying key features of the solutions. Based on the results of the review, the network has been developed. The project addresses recognition with noisy image quality and small learning database. 